\title{
Solving Large-Scale Linear Circuit Problems via Convex Optimization
}

\author{
Javad Lavaei, Aydin Babakhani, Ali Hajimiri and John C. Doyle
}

\begin{abstract}
A broad class of problems in circuits, electromagnetics, and optics can be expressed as finding some parameters of a linear system with a specific type. This paper is concerned with studying this type of circuit using the available control techniques. It is shown that the underlying problem can be recast as a rank minimization problem that is NP-hard in general. In order to circumvent this difficulty, the circuit problem is slightly modified so that the resulting optimization becomes convex. This interesting result is achieved at the cost of complicating the structure of the circuit, which introduces a trade-off between the design simplicity and the implementation complexity. When it is strictly required to solve the original circuit problem, the elegant structure of the proposed rank minimization problem allows for employing a celebrated heuristic method to solve it efficiently.
\end{abstract}

\section{INTRODUCTION}

A vast majority of problems in circuits, electromagnetics, and optics can be regarded as the analysis and synthesis of linear systems in the frequency domain. These systems, in the circuit theory, consist of passive elements including resistors, inductors, capacitors, ideal transformers, and ideal gyrators [1]. Since the seminal work [2], there has been remarkable progress in characterizing such passive (dissipative) systems using the concept of positive real functions. This notion plays a vital role not only in circuit design but also in various control problems [1], [3], [4].

The application of control theory in circuit and communication areas evidently goes beyond the passivity concept. Indeed, the emerging optimization tools developed by control theorists, such as linear matrix inequalities (LMIs) [5] and sum-of-squares (SOS) [6], have been successfully applied to a number of fundamental problems in these fields. For instance, the recent paper [7] proposes an LMI optimization to check whether a given multi-port network can be realized using a pre-specified set of linear time-invariant components (namely an inductor and small-signal model of a transistor). Moreover, the work [8] formulates the pattern synthesis of large arrays with bound constraints on the sidelobe and mainlobe levels as a semidefinite program.

It is well-known that a broad class of problems in circuits, electromagnetics, and optics can be formulated as an optimization over the parameters of a multi-port passive network which is obtained, for instance, via an electromagnetic (EM) simulation. As an example, it is shown in [9] that a strikingly efficient and practical way to deal with certain complex

Javad Lavaei and John C. Doyle are with the Department of Control and Dynamical Systems, California Institute of Technology (emails: lavaei@cds.caltech.edu, doyle@cds.caltech.edu).

Aydin Babakhani and Ali Hajimiri are with the Department of Electrical Engineering, California Institute of Technology, (emails: aydin@caltech.edu, hajimiri@caltech.edu). antenna problems is to extract a circuit model and then search for appropriate values of its parameters. The circuit model proposed in [9] is indeed a simple, general model which could be considered the abstract model of different types of problems. A question arises as to whether there exists a systematic method to study such circuit problems. This paper basically aims to address this question using the available techniques developed in the control theory, especially the LMI and passivity concepts.

Motivated by the work [9], a general circuit problem is considered in this paper, which requires finding a set of parameters to satisfy some prescribed design specifications. It is shown that this problem amounts to solving a simple rank-minimization problem, which is known to be NP-hard in general. Afterwards, the circuit problem is slightly modified to make the resulting optimization problem convex. The convexity proof provided here mainly relies on the elegant properties of passive networks and the power of LMI techniques. The modification made in the circuit problem does not noticeably alter the original circuit or electromagnetic problem, but makes its implementation harder in practice. For this reason, the heuristic method proposed in [10] (and further studied in [11]) is subsequently applied to the obtained rank-minimization problem. It is observed that this heuristic method works satisfactorily, to a great extent.

The paper is organized as follows. In section II, an indepth discussion is provided to outline the deficiencies of the current techniques used to study an electromagnetic problem. Following this motivation, the main results are developed in Section III. Simulation results are then given in Section IV to demonstrate the efficacy of this work.

\section{MOTIVATION AND PROBLEM FORMULATION}

This section first highlights the advantages of solving electromagnetic problems based on their circuit models, and then formalizes the objective of the present work.

Indeed, numerical methods and efficient optimization techniques, enabled by increasing computational power, have been markedly instrumental in advancing the field of modern electrodynamics. The progress in this field which was limited to the development of analytical models for antenna characteristics such as pattern, efficiency, and impedance, has been greatly influenced by novel numerical techniques in time or frequency domains. Frequency domain techniques such as finite element method [12] and method of moments [13], as well as time domain algorithms such as finite difference technique [14], have been extensively used in designing electromagnetic structures. These numerical methods combined with optimization techniques such as genetic algorithm [15] 
and particle swarm optimization [16] provide a valuable, but inefficient, tool in designing large-scale electromagnetic structures where thousands of passive elements are involved. More precisely, the available numerical techniques iteratively search for a sub-optimal solution. Since a new time-consuming EM simulation needs to be run at each iteration, this approach could be really prohibitive, due to the exponential number of iterations. In the recent paper [9], this crucial issue is partially resolved by introducing a novel method, which requires performing the EM simulation only once to extract the scattering parameters of the system. Having obtained this circuit model, the elctromagnetic problem reduces to solving a non-iterative optimization problem over the parameters of a circuit.

The circuit model of the above-mentioned problem and many other ones can be regarded as the circuit given in Figure 1a, in which there are $n$ output ports as follows:

- Output ports $1,2, \ldots, z$ : These ports are the output ports of interest whose voltages must be within some specific ranges.

- Output ports $z+1, z+2, \ldots, n$ : These ports are those output ports which can be controlled by being connected to any passive device.

The objective is to control the output ports $z+1, z+2, \ldots, n$ in such a way that the input impedance at port $n+1$ and the output voltages at ports $1,2, \ldots, z$ lie within specific ranges. The most convenient way of controlling the ports $z+1, z+2, \ldots, n$ is to connect each of them to an ideal switch (as shown in the figure). The question can then be rephrased as whether it is possible to connect a number of the switches in Figure 1a so that some linear constraints on the input impedance and the voltages at the designated output ports are all satisfied. As an alternative, one can use a set of conductances in lieu of the switches (as visualized in Figure $1 \mathrm{~b}$ ), which gives rise to finding the proper values of the conductances. Roughly speaking, every linear problem governed by Maxwells differential equations that requires finding optimal values of the termination impedances may be converted to a similar circuit problem.

\section{MAIN RESULTS}

\section{A. Switching circuit optimization}

To analyze the circuit given in Figure 1a, define the currents $i_{1}, i_{2}, \ldots, i_{n+1}$ and the voltages $v_{1}, v_{2}, \ldots, v_{n+1}$ as shown in the figure. For simplicity, introduce the shorthand notations:

$$
\begin{aligned}
\tilde{\mathbf{i}} & =\left[\begin{array}{llll}
i_{1} & i_{2} & \cdots & i_{n}
\end{array}\right] \\
\tilde{\mathbf{v}} & =\left[\begin{array}{llll}
v_{1} & v_{2} & \cdots & v_{n}
\end{array}\right]
\end{aligned}
$$

One can write two sets of equations as follows:

$$
\begin{aligned}
{\left[\begin{array}{cc}
\tilde{\mathbf{i}} & i_{n+1}
\end{array}\right] } & =\left[\begin{array}{ll}
\tilde{\mathbf{v}} & v_{n+1}
\end{array}\right] Y_{s} \\
i_{n+1} & =y_{\text {in }} \cdot v_{n+1}
\end{aligned}
$$

where:

- $Y_{s}$ is the given $Y$-parameter matrix of a reciprocal, passive network at a specific frequency $\omega_{0}$.
- $v_{n+1}$ is equal to the input voltage $v_{\text {in }}$.

- $y_{\text {in }}$ is the input admittance.

It is worth noting that $Y_{s}$ is a complex-valued matrix whose real and imaginary parts are both symmetric. With no loss of generality, assume that $v_{\text {in }}$ is equal to 1 (this can be achieved after an appropriate scaling, if necessary). The main goal of this part is to address the following problem.

Problem 1: Find whether it is possible to turn on a subset of the switches $\{z+1, z+2, \ldots, n\}$ so that the following constraints are all satisfied:

$$
\begin{aligned}
& \left|\operatorname{Re}\left\{v_{p}-v_{p}^{d}\right\}\right| \leq \varepsilon_{p}, \quad p=1,2, \ldots, z \\
& \left|\operatorname{Im}\left\{v_{p}-v_{p}^{d}\right\}\right| \leq \bar{\varepsilon}_{p}, \quad p=1,2, \ldots, z \\
& \left|\operatorname{Re}\left\{y_{\text {in }}-y_{\text {in }}^{d}\right\}\right| \leq \varepsilon, \\
& \left|\operatorname{Im}\left\{y_{\text {in }}-y_{\text {in }}^{d}\right\}\right| \leq \bar{\varepsilon}
\end{aligned}
$$

where:

- $v_{1}^{d}, \ldots, v_{z}^{d}$ are given desired voltages at the output ports $1, \ldots, z$, respectively.

- $y_{\text {in }}^{d}$ is the desired input admittance.

- Nonnegative numbers $\varepsilon_{p}, \bar{\varepsilon}_{p}, \forall p=1,2, \ldots, z$, and $\varepsilon, \bar{\varepsilon}$ are given permissible tolerances.

Note that the operators $\operatorname{Re}\{\cdot\}$ and $\operatorname{Im}\{\cdot\}$ return the real and imaginary parts of a complex number. Define $\left\{e_{1}, e_{2}, \ldots, e_{n}\right\}$ and $\left\{\tilde{e}_{1}, \tilde{e}_{2}, \ldots, \tilde{e}_{n+1}\right\}$ to be the sets of standard basis vectors of $\Re^{n}$ and $\Re^{n+1}$, respectively. Denote also the set of complex number with $\mathbf{C}$. Throughout this paper, the notations $\succ$ and $\succeq$ will be used to show inequalities in the positive definite and positive semi-definite senses, respectively. The following theorem recasts Problem 1 as an optimization problem.

Theorem 1: Minimize the rank of the matrix:

$$
\left[\begin{array}{c}
X \\
{\left[\begin{array}{cc}
\tilde{\mathbf{v}} & 1
\end{array}\right]}
\end{array}\left[\begin{array}{c}
\tilde{\mathbf{v}}^{*} \\
1 \\
1
\end{array}\right]\right]
$$

for the variables $X \in \mathbf{C}^{(n+1) \times(n+1)}$ and $\tilde{\mathbf{v}} \in \mathbf{C}^{1 \times n}$ subject to the constraints:

$$
\begin{aligned}
& \left|\operatorname{Re}\left\{\tilde{\mathbf{v}} e_{p}-v_{p}^{d}\right\}\right| \leq \varepsilon_{p}, \quad p=1,2, \ldots, z \\
& \left|\operatorname{Im}\left\{\tilde{\mathbf{v}} e_{p}-v_{p}^{d}\right\}\right| \leq \bar{\varepsilon}_{p}, \quad p=1,2, \ldots, z \\
& \left|\operatorname{Re}\left\{\left[\begin{array}{ll}
\tilde{\mathbf{v}} & 1
\end{array}\right] Y_{s} \tilde{e}_{n+1}-y_{\text {in }}^{d}\right\}\right| \leq \varepsilon \\
& \left|\operatorname{Im}\left\{\left[\begin{array}{ll}
\tilde{\mathbf{v}} & 1
\end{array}\right] Y_{s} \tilde{e}_{n+1}-y_{\text {in }}^{d}\right\}\right| \leq \bar{\varepsilon} \\
& {\left[\begin{array}{ll}
\tilde{\mathbf{v}} & 1
\end{array}\right] Y_{s} \tilde{e}_{p}=0, \quad p=1,2, \ldots, z} \\
& X_{q} Y_{s} \tilde{e}_{q}=0, \quad q=z+1, \ldots, n \\
& X=X^{*}
\end{aligned}
$$

where $X_{q}$ is the $q$-th row of the matrix $X$. If the value of the minimum rank is greater than 1, then Problem 1 is not feasible; otherwise, it has a solution which can be extracted from $\tilde{\mathbf{v}}$ by searching for zero entries in this vector and turning on the corresponding switches in the system accordingly.

Proof of necessity: Assume that Problem 1 has a feasible solution, with the output voltage vector $\tilde{\mathbf{v}}$. Given $q \in\{z+$ $1, z+2, \ldots, n\}$, since the ideal switch $q$ has either a zero 


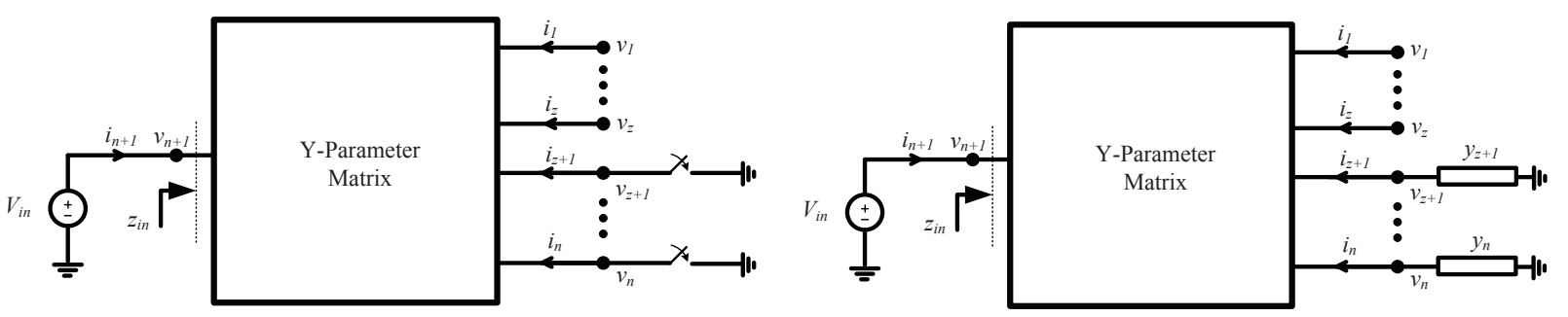

(a)

(b)

Fig. 1. (a): Circuit model with ideal switches; (b): circuit model with conductances.

current or a zero voltage, the product $v_{q}^{*} i_{q}$ is equal to zero. Therefore, it follows from (2a) that:

$$
v_{q}^{*}\left[\begin{array}{cc}
\tilde{\mathbf{v}} & 1
\end{array}\right] Y_{s} \tilde{e}_{q}=0, \quad q=z+1, \ldots, n
$$

On the other hand, since $i_{p}$ is equal to zero for all $p \in$ $\{1,2, \ldots, z\}$, one can write:

$$
\left[\begin{array}{ll}
\tilde{\mathbf{v}} & 1
\end{array}\right] Y_{s} \tilde{e}_{p}=0, \quad p=1,2, \ldots, z
$$

Define $X$ to be:

$$
X:=\left[\begin{array}{c}
\tilde{\mathbf{v}}^{*} \\
1
\end{array}\right]\left[\begin{array}{ll}
\tilde{\mathbf{v}} & 1
\end{array}\right]
$$

Observe that the matrix $X$ defined above, together with the vector $\tilde{\mathbf{v}}$, satisfies all constraints given in (5), i.e., $(X, \tilde{\mathbf{v}})$ is a feasible solution of the optimization problem given in the statement of Theorem 1 for which the rank of the matrix in (4) is equal to 1 . More precisely:

- The conditions $(5 \mathrm{a}),(5 \mathrm{~b}),(5 \mathrm{c}),(5 \mathrm{~d})$ correspond to those given in (3) (by virtue of (2)).

- The condition (5e) is the same as the one given in (7).

- The relation (5f) holds in light of (6) and (8).

- The condition $(5 \mathrm{~g})$ follows immediately from the symmetry of the matrix $X$.

- The rank of the matrix provided in (4) is equal to 1 due to the fact that this matrix can be expressed as the product of two vectors.

Proof of sufficiency: Assume that there exist two matrices $X$ and $\tilde{\mathbf{v}}$ for which the conditions pointed out in the statement of the theorem are all fulfilled. Due to the symmetry of the matrix $X$, it is straightforward to show that there exists a vector $u \in \mathbf{C}^{n+2}$ such that the matrix given in (4) is equal to $u u^{*}$ or $-u u^{*}$ (note that the rank of this matrix is 1 , by assumption). On the other hand, the last diagonal entry of the matrix (4), which is equal to 1 , does not allow this matrix to be negative semi-definite and thus of the form $-u u^{*}$. Hence:

$$
\left[\begin{array}{c}
X \\
{\left[\begin{array}{cc}
\tilde{\mathbf{v}} & 1
\end{array}\right]}
\end{array}\left[\begin{array}{c}
\tilde{\mathbf{v}}^{*} \\
1 \\
1
\end{array}\right]\right]=u u^{*}
$$

This relation can be simplified to conclude that:

$$
\left[\begin{array}{lll}
\tilde{\mathbf{v}} & 1 & 1
\end{array}\right]= \pm u^{*}
$$

As a result, $X$ satisfies the equality:

$$
X=\left[\begin{array}{c}
\tilde{\mathbf{v}}^{*} \\
1
\end{array}\right]\left[\begin{array}{ll}
\tilde{\mathbf{v}} & 1
\end{array}\right]
$$

The rest of the proof relies on this equation and can be carried out in line with the arguments made in the proof of necessity. The details are omitted here for brevity.

Theorem 1 states that checking the feasibility of Problem 1 amounts to solving an optimization problem whose constraints are all linear. However, the rank of a Hermitian matrix is to be minimized, which makes the problem nonconvex. Since a rank-minimization problem is NP-hard in general, there is no efficient algorithm to solve it exactly. The possibility of using a heuristic method to solve this problem will be later discussed. A question arises as to whether Problem 1 can be recast as another optimization problem which can be solved efficiently using deterministic algorithms (rather than non-deterministic or heuristic ones). We showed in [24] that Problem 1 is NP-complete (see Theorem 4 therein), which makes it one of the hardest problems from the computational point of view. Therefore, the goal is to study how Problem 1 can be modified slightly so that it becomes convex. This is the crux of the next subsection.

\section{B. Passive circuit optimization}

Consider again the circuit depicted in Figure 1a. The nonconvexity of Problem 1 originates from the fact that the output ports $z+1, z+2, \ldots, n$ are controlled by ideal switches. In this part, it is desired to allow these ports to be controlled by a general reciprocal passive network as shown in Figure 2. The problem now reduces to finding this network in such a way that the design specifications listed in (3) are satisfied. Let the objective be formalized.

Problem 2: Find whether there exists a reciprocal passive network (as shown in Figure 2) with an admittance $Y$ at the given frequency $\omega_{0}$ for which the following design specifications are met:

$$
\begin{aligned}
& \left|\operatorname{Re}\left\{v_{p}-v_{p}^{d}\right\}\right| \leq \varepsilon_{p}, \quad p=1,2, \ldots, z \\
& \left|\operatorname{Im}\left\{v_{p}-v_{p}^{d}\right\}\right| \leq \bar{\varepsilon}_{p}, \quad p=1,2, \ldots, z \\
& \left|\operatorname{Re}\left\{y_{\text {in }}-y_{\text {in }}^{d}\right\}\right| \leq \varepsilon, \\
& \left|\operatorname{Im}\left\{y_{\text {in }}-y_{\text {in }}^{d}\right\}\right| \leq \bar{\varepsilon}
\end{aligned}
$$




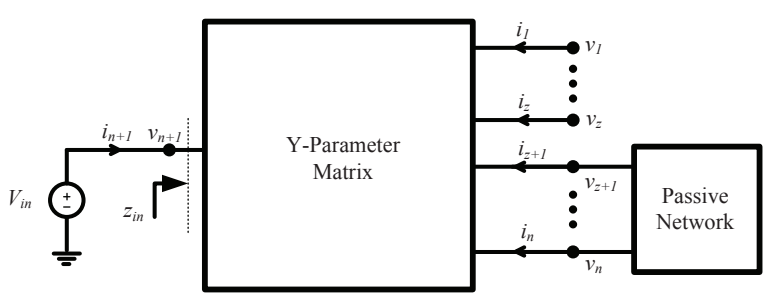

Fig. 2. A modified version of the circuit given in Figure 1a in which a passive network takes the place of the switches.

Note that the reciprocity condition in the above problem can be translated as the real and imaginary parts of $Y$ are both symmetric. It is aimed to show that Problem 2 can be turned into a convex optimization problem of a simple form. To this end, assume that the $Y$-parameter matrix $Y_{s}$ corresponds to a strictly passive network at the frequency $\omega_{0}$. In what follows, a lemma is presented which will be used later to prove this interesting result.

Lemma 1: Given symmetric matrices $M, N \in \Re^{n \times n}$, if $M$ is nonsingular, then the following statements are equivalent:

i) $M$ is a positive definite matrix.

ii) $M+N M^{-1} N$ is a positive definite matrix.

Proof: A proof based on Sylvester's Law of Inertia is provided in the appendix.

Decompose the matrix $Y_{s}$ in a block form as follows:

$$
Y_{s}=\left[\begin{array}{lll}
W_{11} & W_{12} & W_{13} \\
W_{21} & W_{22} & W_{23} \\
W_{31} & W_{32} & W_{33}
\end{array}\right]
$$

where $W_{11} \in \mathbf{C}^{z \times z}, W_{22} \in \mathbf{C}^{(n-z) \times(n-z)}$ and $W_{33} \in \mathbf{C}$. For given symmetric square matrices $A$ and $B$ of the same dimension with $\operatorname{det}(A) \neq 0$, it can be verified that:

$$
\begin{aligned}
(A+B i)^{-1} & =\left(A+B A^{-1} B\right)^{-1} \\
& +\left(A+B A^{-1} B\right)^{-1} B A^{-1} i
\end{aligned}
$$

where $i$ stands for the imaginary unit. This identity will be exploited in the next theorem.

Theorem 2: Problem 2 is feasible if and only if there exist symmetric matrices $M, N \in \Re^{n \times n}$ and vectors $\tilde{\mathbf{v}}_{1} \in \mathbf{C}^{1 \times z}$, $\tilde{\mathbf{v}}_{2} \in \mathbf{C}^{1 \times(n-z)}$ such that:

$$
\left[\begin{array}{cc}
\left(\operatorname{Re}\left\{W_{22}-W_{21} W_{11}^{-1} W_{12}\right\}\right)^{-1}-M & N \\
N & M
\end{array}\right] \succ 0
$$

and:

$$
\begin{aligned}
& \left|\operatorname{Re}\left\{\left[\begin{array}{cc}
\tilde{\mathbf{v}}_{1} & \tilde{\mathbf{v}}_{2}
\end{array}\right] e_{p}-v_{p}^{d}\right\}\right| \leq \varepsilon_{p}, \quad p=1,2, \ldots, z \\
& \left|\operatorname{Im}\left\{\left[\begin{array}{cc}
\tilde{\mathbf{v}}_{1} & \tilde{\mathbf{v}}_{2}
\end{array}\right] e_{p}-v_{p}^{d}\right\}\right| \leq \bar{\varepsilon}_{p}, \quad p=1,2, \ldots, z \\
& \left|\operatorname{Re}\left\{\tilde{\mathbf{v}}_{1} W_{13}+\tilde{\mathbf{v}}_{2} W_{23}+W_{33}-y_{\text {in }}^{d}\right\}\right| \leq \varepsilon \\
& \left|\operatorname{Im}\left\{\tilde{\mathbf{v}}_{1} W_{13}+\tilde{\mathbf{v}}_{2} W_{23}+W_{33}-y_{\text {in }}^{d}\right\}\right| \leq \bar{\varepsilon} \\
& \tilde{\mathbf{v}}_{1}=-\tilde{\mathbf{v}}_{2} W_{21} W_{11}^{-1}-W_{31} W_{11}^{-1} \\
& \tilde{\mathbf{v}}_{2}=\left(W_{31} W_{11}^{-1} W_{12}-W_{32}\right)(M+N i)
\end{aligned}
$$

Moreover, if there exist such matrices $M, N$ satisfying the above constraints, then one candidate for the admittance matrix $Y$ is:

$$
Y=(M+N i)^{-1}-W_{22}+W_{21} W_{11}^{-1} W_{12}
$$

Proof of necessity: Define the vectors:

$$
\begin{aligned}
\tilde{\mathbf{v}}_{1} & =\left[\begin{array}{llll}
v_{1} & v_{2} & \cdots & v_{z}
\end{array}\right] \\
\tilde{\mathbf{v}}_{2} & =\left[\begin{array}{llll}
v_{z+1} & v_{z+2} & \cdots & v_{n}
\end{array}\right]
\end{aligned}
$$

To tackle Problem 2, one can write the following sets of equations:

$$
\begin{aligned}
& {\left[\begin{array}{ll}
\tilde{\mathbf{i}} & i_{n+1}
\end{array}\right]=\left[\begin{array}{lll}
\tilde{\mathbf{v}}_{1} & \tilde{\mathbf{v}}_{2} & 1
\end{array}\right] Y_{s}} \\
& {\left[\begin{array}{ll}
\tilde{\mathbf{i}} & i_{n+1}
\end{array}\right]=-\left[\begin{array}{ccc}
\tilde{\mathbf{v}}_{1} & \tilde{\mathbf{v}}_{2} & 1
\end{array}\right]\left[\begin{array}{ccc}
0 & 0 & 0 \\
0 & Y & 0 \\
0 & 0 & -y_{\text {in }}
\end{array}\right]}
\end{aligned}
$$

These equations can be combined to conclude that:

$$
\begin{array}{r}
\tilde{\mathbf{v}}_{1} W_{11}+\tilde{\mathbf{v}}_{2} W_{21}+W_{31}=0 \\
\tilde{\mathbf{v}}_{1} W_{12}+\tilde{\mathbf{v}}_{2}\left(W_{22}+Y\right)+W_{32}=0 \\
\tilde{\mathbf{v}}_{1} W_{13}+\tilde{\mathbf{v}}_{2} W_{23}+W_{33}-y_{\text {in }}=0
\end{array}
$$

Thus:

$$
\begin{aligned}
& \tilde{\mathbf{v}}_{1}=-\tilde{\mathbf{v}}_{2} W_{21} W_{11}^{-1}-W_{31} W_{11}^{-1} \\
& \tilde{\mathbf{v}}_{2}=\left(W_{31} W_{11}^{-1} W_{12}-W_{32}\right) \tilde{Y} \\
& y_{\text {in }}=\tilde{\mathbf{v}}_{1} W_{13}+\tilde{\mathbf{v}}_{2} W_{23}+W_{33}
\end{aligned}
$$

where:

$$
\tilde{Y}:=\left(W_{22}-W_{21} W_{11}^{-1} W_{12}+Y\right)^{-1}
$$

Note that the invertibility of the the term $W_{22}-$ $W_{21} W_{11}^{-1} W_{12}+Y$ follows from the passivity of $Y$ and the strict passivity of $Y_{s}$. Now, write $\tilde{Y}$ as $M+N i$, for some proper real-valued symmetric matrices $M$ and $N$. It can be verified that the equations (21) and $\tilde{Y}=M+N i$ lead to the equivalence of the constraints given in (16) and those provided in Problem 2. Hence, it remains to show that the passivity of the network with the admittance $Y$ at the frequency $\omega_{0}$ is tantamount to the condition (15). To this end, notice that the passivity constraint on $Y$ can be interpreted as the condition $\operatorname{Re}\{Y\} \succeq 0$ [1]. On applying the identity (14) to the equation (22), this passivity constraint can be expressed as:

$$
\begin{aligned}
\operatorname{Re}\{Y\} & =\operatorname{Re}\left\{\tilde{Y}^{-1}-W_{22}+W_{21} W_{11}^{-1} W_{12}\right\} \\
& =\left(M+N M^{-1} N\right)^{-1} \\
& -\operatorname{Re}\left\{W_{22}-W_{21} W_{11}^{-1} W_{12}\right\} \succeq 0
\end{aligned}
$$

On the other hand, notice that the term $\left(W_{22}-\right.$ $\left.W_{21} W_{11}^{-1} W_{12}\right)^{-1}$ is the $(1,1)$ block entry of the inverse of the matrix:

$$
\left[\begin{array}{ll}
W_{11} & W_{12} \\
W_{21} & W_{22}
\end{array}\right]
$$

which is a principal submatrix of $Y_{s}$. Hence, it follows from the strictly passivity of the admittance matrix $Y_{s}$ that $W_{22}-$ $W_{21} W_{11}^{-1} W_{12}$ corresponds to the impedance of some strictly passive network. This implies that:

$$
\operatorname{Re}\left\{W_{22}-W_{21} W_{11}^{-1} W_{12}\right\} \succ 0
$$


As a result, the passivity constraint given in (23) can be rearranged as:

$$
\left(M+N M^{-1} N\right)^{-1} \succeq \operatorname{Re}\left\{W_{22}-W_{21} W_{11}^{-1} W_{12}\right\} \succ 0
$$

Two properties can be extracted from this relation:

- First, Lemma 1 yields that if the above condition is satisfied, then:

$$
M \succ 0
$$

- Second, the constraint (26) can be manipulated to arrive at:

$$
\operatorname{Re}\left\{W_{22}-W_{21} W_{11}^{-1} W_{12}\right\}^{-1} \succeq M+N M^{-1} N
$$

or equivalently:

$$
\left(\operatorname{Re}\left\{W_{22}-W_{21} W_{11}^{-1} W_{12}\right\}^{-1}-M\right)-N M^{-1} N \succeq 0
$$

The Schur's complement formula asserts that the conditions (27) and (29) are identical to the constraint (15). This completes the proof of necessity.

Proof of sufficiency: The proof can be carried out in line with the above-mentioned argument. The details are omitted for brevity.

Regarding the optimization problem proposed in Theorem 2, it is easy to observe that the constraints are all linear. Therefore, Theorem 2 states that Problem 2 is equivalent to a linear matrix inequality (LMI) feasibility problem, which can be handled efficiently using a proper software tool such as YALMIP or SOSTOOLS [19], [20]. This signifies that replacing switches with a passive network facilitates the circuit design, at the cost of complicating its implementation in practice. In the case when it is strictly required to design a collection of switches, Theorem 2 is still useful. Indeed, infeasibility of Problem 2 implies infeasibility of Problem 1, due to the fact that every switching network is a reciprocal passive network as well. As a result, one can regard the LMI problem proposed in Theorem 2 as a sanity test for checking the feasibility of Problem 1.

Assume that Problem 2 is feasible and, therefore, an admittance matrix $Y$ (at the frequency $\omega_{0}$ ) is obtained by means of solving the feasibility problem given in Theorem 2 . The next step is to design a reciprocal passive (RLC) network whose corresponding admittance transfer function at the frequency $\omega_{0}$ is equal to $Y$. This can be accomplished systematically using the existing methods in the literature [1], [2].

\section{Decoupled passive circuit optimization}

The main issue with the admittance matrix $Y$ obtained in Theorem 2 is that its corresponding RLC network could potentially have so many components, which impede its implementation. To circumvent this drawback, one can impose a sparsity constraint on $Y$, saying that it must be (nearly) diagonal. A diagonal $Y$ converts the circuit model to the one depicted in Figure 1b. It is noteworthy that supplanting an ideal switch with a varactor significantly increases the likelihood that the design specifications (3) be feasible.
Define Problem 3 to be the same as Problem 2, but under the additional constraint of the diagonality of $Y$. It will be shown in the sequel that Problem 3 is non-convex; however, there is a good heuristic method for this problem, as tested on several practical examples.

Theorem 3: Minimize the rank of the matrix:

$$
\left[\begin{array}{ll}
\bar{P} & I \\
I & P
\end{array}\right]
$$

for vectors $\tilde{\mathbf{v}}_{1} \in \mathbf{C}^{1 \times z}, \tilde{\mathbf{v}}_{2} \in \mathbf{C}^{1 \times(n-z)}$, symmetric matrices $M, N \in \Re^{n \times n}$ and diagonal matrices $D_{1}, D_{2} \in \Re^{n \times n}$ subject to the constraints given in (16) and:

$$
\begin{aligned}
D_{1} & \geq 0 \\
M & \succ 0
\end{aligned}
$$

where $P$ and $\bar{P}$ are provided in (39) and (32), respectively. If the value of the minimum rank is greater than $2 n$, then Problem 3 is infeasible; otherwise, it is feasible and a candidate for the diagonal admittance matrix $Y$ can be recovered as follows:

$$
Y=D_{1}+D_{2} i
$$

Proof: When there is no diagonality constraint on the matrix $Y$, a necessary and sufficient condition for the existence of a desirable network is provided in Theorem 2. Hence, it suffices to somehow include this extra constraint. For this purpose, write $Y$ as $D_{1}+D_{2} i$, where $D_{1}$ and $D_{2}$ are required to be diagonal. It results from the equation (17) that:

$$
D_{1}+D_{2} i+W_{22}-W_{21} W_{11}^{-1} W_{12}=(M+N i)^{-1}
$$

Applying the identity (14) to the above equation yields that:

$$
\begin{aligned}
D_{1}+\operatorname{Re}\left\{W_{22}-W_{21} W_{11}^{-1} W_{12}\right\} & =\left(M+N M^{-1} N\right)^{-1} \\
D_{2}+\operatorname{Im}\left\{W_{22}-W_{21} W_{11}^{-1} W_{12}\right\} & =\left(M+N M^{-1} N\right)^{-1} \\
& \times N M^{-1}
\end{aligned}
$$

These equations can be written in the matrix form as the one expressed in (36), or equivalently $\bar{P}=P^{-1}$. On the other hand:

$$
\left[\begin{array}{ll}
\bar{P} & I \\
I & P
\end{array}\right]=\left[\begin{array}{cc}
I & P^{-1} \\
0 & I
\end{array}\right]\left[\begin{array}{cc}
\bar{P}-P^{-1} & 0 \\
0 & P
\end{array}\right]\left[\begin{array}{cc}
I & 0 \\
P^{-1} & I
\end{array}\right]
$$

In light of the equality $\bar{P}-P^{-1}=0$ and the non-singularity of $P$, it can be concluded from the above equation that the rank of the matrix given in (30) is exactly equal to $2 n$. So far, it is shown that the diagonality of the matrix $Y$ implies the aforementioned rank constraint. To prove the converse statement, notice that the condition $M \succ 0$ makes the Hamiltonian matrix $P$ nonsingular (see the proof of Lemma 1). This, together with the identity (37), signifies that if the rank of the matrix in (30) is $2 n$, then the matrix $P-\bar{P}^{-1}$ must be zero. This result leads to the equation (34), which is the diagonality constraint of the matrix $Y$. Moreover, one can easily replace the passivity constraint (15) given in Theorem 2 with the condition $D_{1} \geq 0$, because the real part of the matrix $Y$ is equal to $D_{1}$. This completes the proof. 


$$
\begin{gathered}
\bar{P}:=\left[\begin{array}{cc}
D_{1}+\operatorname{Re}\left\{W_{22}-W_{21} W_{11}^{-1} W_{12}\right\} & -D_{2}-\operatorname{Im}\left\{W_{22}-W_{21} W_{11}^{-1} W_{12}\right\} \\
-D_{2}-\operatorname{Im}\left\{W_{22}-W_{21} W_{11}^{-1} W_{12}\right\} & -D_{1}-\operatorname{Re}\left\{W_{22}-W_{21} W_{11}^{-1} W_{12}\right\}
\end{array}\right] \\
{\left[\begin{array}{cl}
D_{1}+\operatorname{Re}\left\{W_{22}-W_{21} W_{11}^{-1} W_{12}\right\} & -D_{2}-\operatorname{Im}\left\{W_{22}-W_{21} W_{11}^{-1} W_{12}\right\} \\
-D_{2}-\operatorname{Im}\left\{W_{22}-W_{21} W_{11}^{-1} W_{12}\right\} & -D_{1}-\operatorname{Re}\left\{W_{22}-W_{21} W_{11}^{-1} W_{12}\right\}
\end{array}\right]=\left[\begin{array}{cc}
M & N \\
N & -M
\end{array}\right]^{-1}}
\end{gathered}
$$

\section{Heuristic method for rank minimization}

A rank minimization problem is known to be NP-hard in general. However, several heuristic methods have been proposed in the literature to relax the problem to a convex one, whose solution may be identical or near to that of the original problem [10], [18]. In particular, the works [10], [11] suggest minimizing the nuclear norm, i.e., the sum of the singular values, of the matrix whose rank is to be minimized. This heuristic method works correctly with overwhelming probability for a broad class of random rank-minimization problems. Although the rank minimization problems given in Theorems 1 and 3 may not lie into that category of wellbehaved problems, an extensive simulation was done by the authors to test the efficiency of this method over different antenna problems. It was observed that the heuristic method works perfectly for the optimization problem in Theorem 3 if there is only constraints on $y_{\text {in }}$. When there are constraints on the output voltages, some of the constraints may be violated a trifle. This might be partially due to the fact that the circuit problem is not numerically robust with respect to these voltages, and a small change in the parameters of the network may make the voltages alter noticeably. Note that the nuclear-norm heuristic method often fails to obtain a satisfactory result when applied to Theorem 1, as simulation suggests. This is by virtue of the fact that Problem 1 is NPcomplete.

Remark 1: The circuit problem investigated in this paper may need to be under additional constraints as follows:

- The design specifications given in (3) are only at the frequency $\omega_{0}$. There could be multiple frequencies, each one associated with similar design requirements.

- There may be extra conditions on the matrix $Y$ in Problems 2 and 3, such as being lossless.

- Some of the output voltages may be required to be sufficiently weak. This introduces norm inequality constraints.

The optimization results obtained in the paper can be straightforwardly modified to encompass the above constraints, in addition to many others.

\section{Simulation RESUlts}

Let the efficacy of the present work be elucidated in the context of the antenna design. Note that most of the practical antenna problems deal with the optimization of the input impedance and/or the antenna gain via changing the geometry of the antenna. This is achieved in reality by means of generic algorithms. For instance, a particle swarm optimization technique (PSO) is deployed in [25] to optimize the antenna input impedance by varying its length, width, and feeding point. That algorithm was applied to a simple impedance matching problem with only 3 variables, which consumed more than 25 hours to obtain the solution. This clearly shows that such generic algorithms are dramatically time-consuming even for very small-sized antenna problems. The present paper aims to illustrate that a more complicated antenna design problem with 12 variables can be solved in the order of seconds rather than hours using the method developed here.

Consider the antenna configuration depicted in Figure 3, which consists of a transmitting dipole antenna (blue bar), a $3 \times 3$ array of metal plates (antenna parasitic elements), and a receiving dipole antenna located at the far field (green bar). There are 14 ports in this figure as follows:

- Port 1 acts as a receiving antenna sampling the radiation pattern of the transmitting antenna at a specific angle in the far field.

- Ports 2 to 13 are intended to change the boundary condition of the transmitting antenna.

- Port 14 corresponds to the transmitting antenna.

The objective is to find optimum impedance values for the parasitic elements such that the received power and the antenna input impedance satisfy a specific set of constraints. For this purpose, the circuit model of the antenna system is extracted at the desired frequency $3.5 \mathrm{GHz}$ (using localized differential lumped ports) by means of the electromagnetic software IE3D [21]. This model can be any of the circuits given in Figures 1a, 1b, 2, depending on how the impedances of the parasitic elements are designed. Note that $n$ and $z$ are equal to 13 and 1 , respectively, in this example, and that $v_{n+1}=v_{14}=1$.

Three important goals in a typical antenna problem are (i) received power maximization, (ii) received power maximization under an input admittance constraint, (iii) input impedance matching. Tackling these problems is central to this section, which is carried out in the sequel.

Notice that the power at the receiving antenna is proportional to the 2-norm of $v_{1}$ raised to the second power. Since maximizing the 2-norm of any quantity is normally a nonconvex problem, it is desired to maximize the 1-norm of $v_{1}$, i.e. $\left|\operatorname{Re}\left\{v_{1}\right\}\right|+\left|\operatorname{Im}\left\{v_{1}\right\}\right|$. This suggestion is motivated by the close affinity between these two norms. Observe that the direct maximization of $\left|\operatorname{Re}\left\{v_{1}\right\}\right|+\left|\operatorname{Im}\left\{v_{1}\right\}\right|$ is again a nonconvex optimization problem. Nevertheless, one can alternatively perform four (convex) optimizations maximizing the quantities $\operatorname{Re}\left\{v_{1}\right\}+\operatorname{Im}\left\{v_{1}\right\}, \operatorname{Re}\left\{v_{1}\right\}-\operatorname{Im}\left\{v_{1}\right\},-\operatorname{Re}\left\{v_{1}\right\}+$ $\operatorname{Im}\left\{v_{1}\right\}$, and $-\operatorname{Re}\left\{v_{1}\right\}-\operatorname{Im}\left\{v_{1}\right\}$, and then determine the 


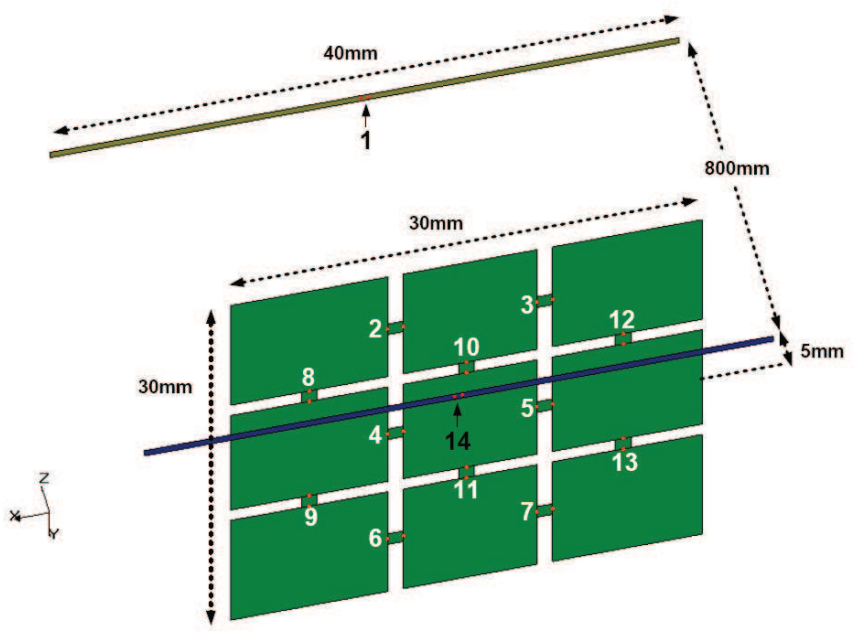

Fig. 3. Antenna problem studied in Section IV.

maximum solution corresponding to the desirable objective function $\left|\operatorname{Re}\left\{v_{1}\right\}\right|+\left|\operatorname{Im}\left\{v_{1}\right\}\right|$. Problem 2 is adapted to solve these optimization problems. The outcome of these convex optimization problems is summarized in Table I, which demonstrates that the optimal value of $\left|\operatorname{Re}\left\{v_{1}\right\}\right|+\left|\operatorname{Im}\left\{v_{1}\right\}\right|$ is equal to 0.2833 that corresponds to the antenna directivity of $8.17 \mathrm{dBi}$ and the radiation efficiency of $89.15 \%$. It is interesting to note that this result is obtained by solving four convex optimization problems, each of which is handled by the software CVX [22] in a fraction of second (the simulation was run on a computer with a Pentium IV $3.0 \mathrm{GHz}$ and 3.62 GB of memory).

Now, assume that the objective is to maximize the power at the receiving antenna subject to the constraint that the antenna input impedance is equal to the standard value $50 \Omega$. As before, this power is proportional to the 2-norm of the output voltage $v_{1}$ raised to the second power. The nonconvexity of the underlying problem suggests maximizing the closely related term $\left|\operatorname{Re}\left\{v_{1}\right\}\right|+\left|\operatorname{Im}\left\{v_{1}\right\}\right|$. Similar to the previous case, four convex optimization problems are solved, and the results are summarized accordingly in Table II.

As the last scenario, the goal is to find a diagonal matrix $Y$ such that the antenna input impedance is matched with the value $50 \Omega$. The heuristic method given in [11] was applied to Problem 3 to find proper values for the diagonal matrices $D_{1}$ and $D_{2}$ (recall that $Y=D_{1}+D_{2} i$ ). Fortunately, an appropriate solution was found as follows:

$$
\begin{gathered}
D_{1}=\operatorname{diag}[0,0.0026,0.0026,0.0070,0.0070,0.0026,0.0026, \\
\quad 0.0138,0.0134,0.3252,0.4268,0.0136,0.0123] \\
D_{2}=\operatorname{diag}[0,-0.0106,-0.0105,-0.0064,-0.0064,-0.0106, \\
\quad-0.0105,0.0215,0.0217,-0.0050,-0.0036, \\
0.0227,0.0205]
\end{gathered}
$$

which corresponds to the antenna directivity of $3.55 \mathrm{dBi}$.

\section{CONCLUSiOnS}

This paper studies a class of linear networks that appear in circuits, electromagnetics, optics, etc. Given such a linear system, the objective is to find certain parameters of the circuit (system) subject to some design specifications. This circuit problem is tantamount to a rank minimization problem of a simple form. In light of the particular structure of this optimization, it is verified that a celebrated heuristic method works satisfactorily for this non-convex optimization. Moreover, it is proved that a slight modification of the underlying problem makes the corresponding optimization problem convex. This modification is pragmatic and solely complicates the device implementation. The results of the current work are derived using available techniques in the control theory.

\section{ACKNOWLEDGMENT}

This research was supported by ONR MURI N00014-081-0747 "Scalable, Data-driven, and Provably-correct Analysis of Networks," ARO MURI W911NF-08-1-0233 "Tools for the Analysis and Design of Complex Multi-Scale Networks," and the Army's W911NF-09-D-0001 Institute for Collaborative Biotechnology.

\section{REFERENCES}

[1] N. Nagai, "Linear circuits, systems, and signal processing: advanced theory and applications," Marcel Dekker, 1990.

[2] O. Brune, "Synthesis of a finite two terminal network whose drivingpoint impedance is a prescribed function of frequency," Journal of Mathematics and Physics, vol. 10, pp. 191-236, 1931.

[3] K. S. Narendra and A. M. Annaswamy, "Stable adaptive systems," Dover, 2005.

[4] J. Bao and P. L. Lee, "Process control: the passive systems approach," Springer, 2007.

[5] S. Boyd and L. Vandenberghe, "Convex Optimization," Cambridge University Press, 2004.

[6] P. A. Parrilo, "Structured semidefinite programs and semialgebraic geometry methods in robustness and optimization," PhD dissertation, California Institute of Technology, 2000. 
TABLE I

MAXIMIZING THE RECEIVED POWER

\begin{tabular}{|c|c|c|c|c|l|}
\hline Objective function & Optimal value & Voltage at port 1 & Directivity & Radiation efficiency & CPU time \\
\hline $\operatorname{Re}\left\{v_{1}\right\}+\operatorname{Im}\left\{v_{1}\right\}$ & 0.0491 & $-0.0126-0.0365 i$ & $7.62 \mathrm{dBi}$ & $84.72 \%$ & $0.34 \mathrm{sec}$ \\
\hline $\operatorname{Re}\left\{v_{1}\right\}-\operatorname{Im}\left\{v_{1}\right\}$ & 0.1423 & $-0.0126+0.1297 i$ & $6.81 \mathrm{dBi}$ & $93.4 \%$ & $0.55 \mathrm{sec}$ \\
\hline$-\operatorname{Re}\left\{v_{1}\right\}+\operatorname{Im}\left\{v_{1}\right\}$ & 0.1902 & $0.1536-0.0365 i$ & $7.97 \mathrm{dBi}$ & $85.41 \%$ & $0.56 \mathrm{sec}$ \\
\hline$-\operatorname{Re}\left\{v_{1}\right\}-\operatorname{Im}\left\{v_{1}\right\}$ & 0.2833 & $0.1536+0.1297 i$ & $8.17 \mathrm{dBi}$ & $89.15 \%$ & $0.63 \mathrm{sec}$ \\
\hline
\end{tabular}

TABLE II

MAXIMIZING THE RECEIVED POWER AFTER IMPOSING IMPEDANCE CONSTRAINT AT THE INPUT OF THE ANTENNA

\begin{tabular}{|c|c|c|c|c|l|}
\hline Objective function & Optimal value & Voltage at port 1 & Directivity & Radiation efficiency & CPU time \\
\hline $\operatorname{Re}\left\{v_{1}\right\}+\operatorname{Im}\left\{v_{1}\right\}$ & 0.0432 & $-0.0192-0.0240 i$ & $7.34 \mathrm{dBi}$ & $86.472 \%$ & $0.78 \mathrm{sec}$ \\
\hline $\operatorname{Re}\left\{v_{1}\right\}-\operatorname{Im}\left\{v_{1}\right\}$ & 0.0579 & $-0.0192+0.0387 i$ & $6.30 \mathrm{dBi}$ & $90.15 \%$ & $0.87 \mathrm{sec}$ \\
\hline$-\operatorname{Re}\left\{v_{1}\right\}+\operatorname{Im}\left\{v_{1}\right\}$ & 0.0674 & $0.0434-0.0240 i$ & $6.56 \mathrm{dBi}$ & $85.58 \%$ & $0.86 \mathrm{sec}$ \\
\hline$-\operatorname{Re}\left\{v_{1}\right\}-\operatorname{Im}\left\{v_{1}\right\}$ & 0.0821 & $0.0434+0.0387 i$ & $7.75 \mathrm{dBi}$ & $89.3 \%$ & $0.82 \mathrm{sec}$ \\
\hline
\end{tabular}

[7] J. Harrison, "Formal synthesis of circuits with minimum noise figure using linear matrix inequalities," IEEE Transactions on Circuits and Systems, vol. 54, no. 4, pp. 855-862, 2007.

[8] H. G. Hoang, H. D. Tuan and B. N. Vo, "Low-dimensional SDP formulation for large antenna array synthesis," IEEE Transactions on Antennas and Propagation, vol. 55, no. 6, pp. 1716-1725, 2007.

[9] A. Babakhani, D. B. Rutledge and A. Hajimiri, "Transmitter architectures based on near-field direct antenna modulation," IEEE Journal of Solid-State Circuits, vol. 43, no. 12, pp. 2674-2692, 2008.

[10] M. Fazel, H. Hindi and S. Boyd, "A rank minimization heuristic with application to minimum order system approximation," American Control Conference, Arlington, Virginia, 2001.

[11] B. Recht, M. Fazel and P. A. Parrilo, "Guaranteed minimum rank solutions to linear matrix equations via nuclear norm minimization," submitted to SIAM Review, 2007.

[12] J. M. Jin, "The finite element method in electromagnetics," New York: Wiley, 1993.

[13] R. F. Harrington, "Field computation by moment methods," Piscataway, NJ: IEEE Press, 1993.

[14] K. S. Kunz and R. J. Luebbers, "The finite difference method for electromagnetics," CRC Press, London, 1993.

[15] Y. Rahmat-Samii and E. Michielssen, "Electromagnetic optimization by genetic algorithms," Wiley \& Sons, New York, 1999.

[16] J. Robinson and Y. Rahmat-Samii, "Particle swarm optimization in electromagnetics," IEEE Transactions on Antennas and Propagation, vol. 52, no. 2, pp. 397-407, 2004.

[17] B. K. Natarajan, "Sparse approximate solutions to linear systems," SIAM Journal of Computing, vol. 24, no. 2, pp. 227-234 , 1995.

[18] K. M. Grigoriadis and E. B. Beran, "Alternating projection algorithms for linear matrix inequalities problems with rank constraints," $A d$ vances in Linear Matrix Inequality Methods in Control, SIAM, 2000.

[19] J. Löfberg, "A toolbox for modeling and optimization in MATLAB," in Proceedings of the CACSD Conference, Taipei, Taiwan, 2004.

[20] S. Prajna, A. Papachristodoulou, P. Seiler and P. A. Parrilo, "SOSTOOLS sum of squares optimization toolbox for MATLAB," Users guide, 2004.

[21] IE3D electromagnetic simulation and optimization software, Zeland Software Inc., www.zeland.com.

[22] M. Grant and S. Boyd, "Matlab software for disciplined convex programming (web page and software)," http://stanford.edu/ boyd/cvx, 2009.

[23] B. Hayes, "Group theory in the bedroom, and other mathematical diversions," Hill \& Wang, 2008.

[24] J. Lavaei, A. Babakhani, A. Hajimiri and J. C. Doyle, "Solving large-scale linear circuit problems via convex optimization," Technical Report, California Institute of Technology, 2009 (available at www.cds.caltech.edu/ lavaei/Tech_rep2_2009.pdf).

[25] N. Jin and Y. Rahmat-Samii, "Parallel particle swarm optimization and finite-difference time-domain (PSO/FDTD) algorithm for multiband and wide-band patch antenna designs, IEEE Transactions on Antenna and Propagation, vol. 53, no. 11, pp. 34593468, 2005.

\section{APPENDIX}

Proof of Lemma 1: It is evident that (i) implies (ii). Therefore, it only remains to prove the converse statement. To this end, assume that $M+N M^{-1} N$ is a positive definite matrix. Define the following matrices:

$$
\begin{aligned}
P & :=\left[\begin{array}{cc}
M & N \\
N & -M
\end{array}\right], \\
T & :=\left[\begin{array}{cc}
I & -N M^{-1} \\
0 & I
\end{array}\right], \\
Q & :=\left[\begin{array}{cc}
M+N M^{-1} N & 0 \\
0 & -M
\end{array}\right]
\end{aligned}
$$

It is easy to verify that $P=T Q T^{*}$. Denote the number of positive, negative and zero eigenvalues of the symmetric matrix $P$ with $\eta_{1}, \eta_{2}, \eta_{3}$, respectively. In the same way, denote the same quantities of the matrix $Q$ with the triple $\left(\bar{\eta}_{1}, \bar{\eta}_{2}, \bar{\eta}_{3}\right)$. Since the matrix $T$ is nonsingular, it follows from Sylvester's Law of Inertia that:

$$
\left(\eta_{1}, \eta_{2}, \eta_{3}\right)=\left(\bar{\eta}_{1}, \bar{\eta}_{2}, \bar{\eta}_{3}\right)
$$

On the other hand, the Hamiltonian structure of the matrix $P$ concludes that:

$$
\eta_{1}=\eta_{2}
$$

Furthermore, since the matrix $M+N M^{-1} N$ is positive definite, $\bar{\eta}_{1}$ is at least equal to $n$, i.e., the size of this matrix. In light of the equalities (40) and (41), this is possible only when $\eta_{1}=\eta_{2}=\bar{\eta}_{1}=\bar{\eta}_{2}=n$. Thus, the matrix $Q$ has $n$ negative eigenvalues. Nonetheless, the negative eigenvalues of this matrix are the same as those of the matrix $-M$; hence, $-M \in \Re^{n \times n}$ has the maximum number of negative eigenvalues. This simply proves that the eigenvalues of $M$ are all positive, which completes the proof. 